\title{
Severe hypotony and filtering bleb leak after intravitreal injection of ranibizumab
}

\author{
Ilias Georgalas \\ Dimitris Papaconstantinou \\ loannis Tservakis \\ Chrysanthi Koutsandrea \\ loannis Ladas \\ "G.Gennimatas" Hospital of Athens, \\ NHS, Athens, Greece
}

\begin{abstract}
We report a case of a patient with age-related macular degeneration, who had undergone trabeculectomy nine years earlier, and developed severe hypotony and bleb leak after treatment with intravitreal injections of ranibizumab. Although the incident is rare, in patients with longstanding filtering blebs, who need treatment with antivascular endothelial growth factors, close follow-up may be advocated, since the occurrence of bleb rupture could lead to permanent vision loss.
\end{abstract}

Keywords: trabeculectomy, ranibizumab, age-related macular degeneration

\section{Introduction}

Trabeculectomy was introduced by Cairns in 1968 and until today is the most common surgical procedure for the treatment of glaucoma. ${ }^{1,2}$ The procedure is supposed to remove a portion of trabecular meshwork to allow flow into the cut ends of Schlemm's canal, using a partial thickness scleral flap to cover the sclerostomy; ${ }^{1,2}$ and thus a fistula is created between the anterior chamber and the subjuconjunctival space.

We report case of a patient with age-related macular degeneration (AMD) who had undergone trabeculectomy nine years earlier and developed severe hypotony and bleb leak after injection with antivascular endothelial growth factor (VEGF) which has never been reported before to the best of our knowledge.

\section{Case presentation}

A 71-year-old man was referred to us with the diagnosis of exudative maculopathy due to AMD in his right eye. He denied a history of systemic illness, eye injury, use of any medication, apart from diabetes mellitus. The patient was a nonsmoker.

Nine years ago, he had undergone uncomplicated trabeculectomy without any adjuncts for the treatment of primary open angle glaucoma. His intraocular pressure (IOP) had been within normal limits without any additional medication, post trabeculectomy. Four years after trabeculectomy, he underwent uncomplicated phacoemulsification and his visual acuity (VA) was recorded to be 20/30 a month after surgery.

On examination VA of the right eye was 20/800 and fundus examination revealed a macular elevation and submacular hemorrhage; Fluorescein angiography depicted a predominantly classic subfoveal choroidal neovascularization (CNV) in the right eye, and the patient was diagnosed as having exudative AMD. After a discussion about the risks and benefits of intravitreal anti-VEGF therapy, the patient consented to treatment and received two intravitreal injections of ranibizumab (Lucentis) with a one-month interval. After each intravitreal injection G. Tobramycin qid was prescribed, for three days.

Three weeks after the second injection of Lucentis, the patient presented with discomfort, tearing, and blurred vision. Visual acuity was "light perception", IOP 
was $2 \mathrm{mmHg}$, and cornea was edematous with descemet folds (Figure 1). Seidel test was positive, indicating leakage from the thinned bled.

The patient was taken to theatre where the above findings were confirmed and a vitreous strand coming out of the ruptured bleb was noted. During surgery careful dissection of the vitreous from the surgical wound was performed (Figure 2) and the gap of tissue in sclerostomy site was covered with human pericardium; subsequently conjunctiva was sutured above it.

The next day after surgery, the anterior chamber was well formed, IOP was $10 \mathrm{mmHg}$ and VA improved to "counting fingers". Six weeks later, Seidel test was negative, the IOP was $12 \mathrm{mmHg}$ and the retina attached.

\section{Discussion}

Age-related macular degeneration is the most common cause of visual loss in patients over 65 years of age. ${ }^{3,4}$ Neovascular AMD with the development of $\mathrm{CNV}$ in the macular area accounts for $80 \%$ of the severe loss of visual acuity due to AMD ${ }^{3,4}$ Ranibizumab is a recombinant humanized immunoglobulin G1 and isotype monoclonal antibody fragment designed for intraocular use which binds to and inhibits the biologic activity of human vascular endothelial growth factor A. The latter is believed to contribute to the development and/or progression of choroidal neovascularization associated with neovascular (wet) AMD. ${ }^{5,6}$ Results of the MARINA study and many other reports support the favourable results of ranibizumab. ${ }^{6}$

Recently, another anti-VEGF, bevacizumab, has been used as an adjunct for reducing the incidence of bleb failure

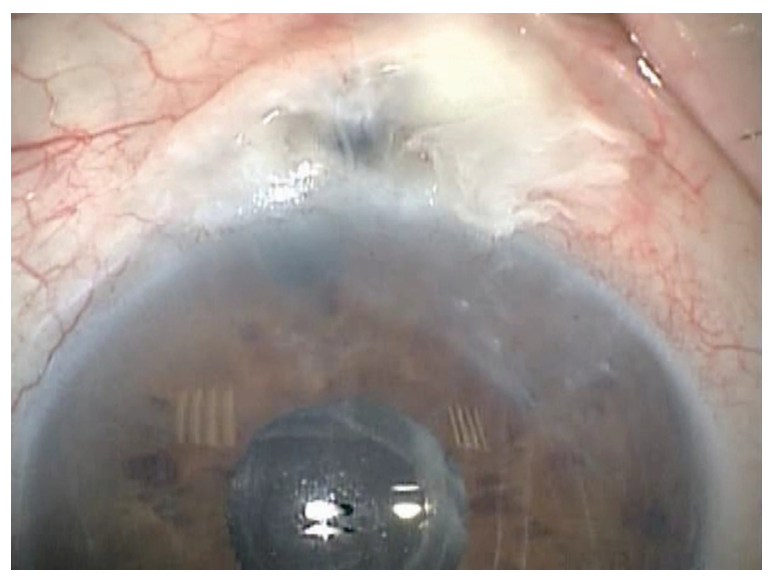

Figure I At presentation; note the extremely thin and avascular bleb and the descemet folds in the cornea secondary to hypotony.

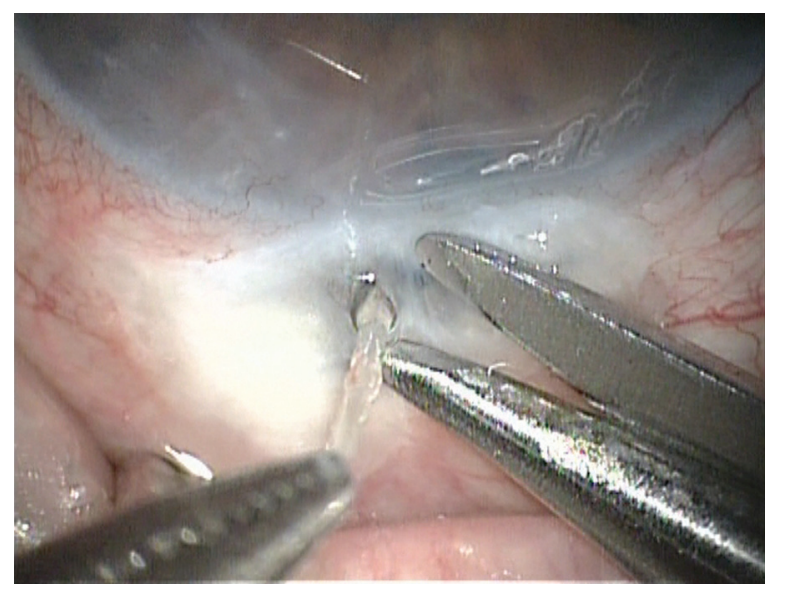

Figure 2 In theatre; note the vitreous strands coming out of the sclerostomy site.

after trabeculectomy ${ }^{7,8}$ and the six-month outcomes are encouraging. ${ }^{7}$

In our case, we postulate that the anti-VEGF intravitreal as an angiogenesis inhibitor, ranibizumab, prevented the formation of new blood vessels, resulting in a lack of supply of oxygen and nutrients to the tissues. This may have resulted in ischemia and necrosis (consequently inhibiting reversal of the process and impairing wound healing) which led to the bleb leak and the severe hypotony in our patient. The fact that our patient was diabetic should be also taken into consideration. Coote and colleagues reported that a single subconjunctival bevacizumab injection led to a dramatic reduction in bleb vascularity for six weeks. ${ }^{8}$ Similarly, in our patient, the reduction of the bleb vascularity may have led to gradual thinning and finally to bleb rupture.

It is highly unlikely that the latter is due to chance since the filtering surgery had been performed nine years previously. The bleb leak occurred three weeks after the second injection of Lucentis and this could be attributed to the fact that it takes time for an anti-VEGF to shut down the existing vessels that supply the bleb, leading to ischemia and necrosis.

On the other hand wound-healing problems have been reported after systemic use of bevacizumab, ${ }^{9}$ however, in surgical oncology there is little evidence to link anastomotic leak with bevacizumab use. ${ }^{10}$

In conclusion, although the incident is rare and data from one patient cannot establish a cause effect relationship between treatment and side-effects, in patients with longstanding filtering blebs that need anti-VEGF treatment, a closer follow up of the patient could be considered, since the occurrence of bleb rupture could lead to permanent vision loss. 


\section{Disclosure}

The authors report no conflicts of interest in this work.

\section{References}

1. Watson PG. Trabeculectomy. Dev Ophthalmol. 1981;1:61-70.

2. Cairns JE. Trabeculectomy. Preliminary report of a new method. Am J Ophthalmol. 1968;66:673-679.

3. Ambati J, Ambati BK, Yoo SH, Ianchulev S, Adamis AP. Age-related macular degeneration: etiology, pathogenesis, and therapeutic strategies. Surv Ophthalmol. 2003;48:257-293.

4. Bressler NM, Bressler SB, Fine SL. Age-related macular degeneration. Surv Ophthalmol. 1988;32:375-413.

5. Gaudreault J, Fei D, Rusit J, Suboc P, Shiu V. Preclinical pharmacokinetics of Ranibizumab (rhuFabV2) after a single intravitreal administration. Invest Ophthalmol Vis Sci. 2005;46:726-733.
6. Chang TS, Bressler NM, Fine JT, Dolan CM, Ward J, Klesert TR Improved vision-related function after ranibizumab treatment of neovascular age-related macular degeneration: results of a randomized clinical trial. Arch Ophthalmol. 2007;125:1460-1469.

7. Grewal DS, Jain R, Kumar H, Grewal SP. Evaluation of subconjunctival bevacizumab as an adjunct to trabeculectomy: A pilot study. Ophthalmology. 2008. Aug 7. [Epub ahead of print].

8. Coote MA, Ruddle JB, Qin Q, Crowston JG. Vascular changes after intra-bleb injection of bevacizumab. J Glaucoma. 2008;17:517-518.

9. Scott LJ. Bevacizumab: in first-line treatment of metastatic breast cancer. Drugs. 2007;67:1793-1799.

10. Gruenberger B, Tamandl D, Schueller J, et al. Bevacizumab, capecitabine, and oxaliplatin as neoadjuvant therapy for patients with potentially curable metastatic colorectal cancer. J Clin Oncol. 2008;26:1830-1835. 
